\title{
The Parodontium under Homeostatic Conditions
}

\author{
Ambarkova Vesna* \\ Department for preventive and pediatric dentistry, Faculty of dentistry, University Ss. Cyril \& Methodius, Skopje, Republic of \\ Macedonia
}

Received: June 27, 2018; Accepted: June 06, 2018; Published: June 20, 2018

*Corresponding author: Vesna Ambarkova, University St. Cyril and Methodius, Faculty of Dental Medicine, Department of Paediatric and Preventive Dentistry, Mother Theresa 17 University Dental Clinic Center Sv. Pantelejmon Skopje 1000, Republic of Macedonia, Tel: ++38970686333, E-mail: vesna. ambarkova@gmail.com

\section{Introduction}

The gingival epithelium is the first line of the host defense, physically impeding the microbial invasion of the periodontal tissues, but there is also a biological action to suppress the spread of pathogenic microbes [1]. In the absence of clinical signs of inflammation, about 30,000 polymorphonuclear leucocytes migrate per minute through the gingival epithelium to the bottom of the gingival sulcus, together with different molecules, which is a host defense system against bacterial invasion [2]. There is a dynamic equilibrium in the host cell bacteria, which can be disrupted by increased accumulation of bacteria on the surface of the teeth, or from present factors.

The coordinated regulation of cell proliferation and differentiation are events that are controlled by the signaling mechanisms of the host, and it is a tissue homeostasis. These signaling mechanisms in the maintenance of parodontium homeostasis are carried out by regulating epithelial cellular functions, as well as the functions of connective tissue cells and hematopoietic cells [3]. In the early stage of the disease, the first bacteria-mediated cells are parodontium fibroblasts that relieve cytokines and chemo-attributants that are important regulators of the inflammatory process, as well as factors that directly participate in bone metabolism (from Receptor Activator of Nuclear Factor $\kappa B$ Ligand, RANKL). These cells also secrete molecules involved in cell migration and extracellular matrix degradation, which in turn leads to further leukocyte infiltration and thereby defense against pathogens, but can also lead to tissue damage [4].

The matrix metalloproteinases MMPs are a family of extracellular proteins that participate in tissue remodeling processes in normal and pathological conditions [5]. In physiological terms, the activity of the matrix metalloproteinase MMP is low as a result of strict regulation at the transcriptional and post-transcriptional levels, and is usually found in balance with a group of endogenous proteins, tissue Inhibitors of Metalloproteinases (TIMPs). Imbalance in the matrix metalloproteinase MMP / tissue inhibitors of metalloproteinase TIMP system is present in periodontal disease and in the pathogenesis of several other diseases such as rheumatoid arthritis, which share several other periodontal disease characteristics, and one of them is the chronic nature of the disease [6]. Accordingly, inhibition of tissue inhibitors of metalloproteinase MMP is proposed as adjuvant therapy of periodontal disease [7].

The Periodontal Ligament (PDL) is crucial for positioning teeth in the alveolar bone and absorbing chewing gum forces. The periodontal ligament PDL cells have the ability to multiply, migrate, and synthesize several components of the parodontium, and also participate in both mechanisms - protective and destructive which prevents periodontal disease development or initiates lesions and promotes disease progression by the action of various biological mechanisms. The cells of periodontal ligament PDLc proliferation is considered to be one of the major events in parodontal homeostasis, which depends on maintaining the delicate balance between bone resorption of osteoclasts and bone synthesis from osteoblasts, that is, the balance between RANCL and Osteoprotegerin (OPG). The Osteoprotegerin (OPG) is essential for bone remodeling [8]. With respect to periodontal disease, increased RNA expression is described in studies [9-12].

Homeostasis of the tissue represents the delicate balance between anabolic and catabolic activities. Parodontium and in a healthy mouth, it constantly creates cytokines, chemokines, and mobile adhesion molecules that are associated with a baseline level of inflammation, providing protection against bacterial challenge without resulting tissue damage. Interestingly, some cytokines associated with chronic inflammation and tissue damage, such as interleukin -1(IL-1), interleukin -6 (IL-6), tumor necrosis factor-alpha (TNF-alpha), are found in the gingival cervical fluid from clinically healthy places, but lower in relation to diseased sites. In this context, the transition from a healthy to an inflammatory state appears to be associated with quantitative and qualitative changes in the inflammatory immune response.

Many studies suggest that inflammation as well as hypoxia leads to an increase of $\mathrm{p} 53$ protein levels. However, the implication of p53 during oral inflammatory processes is still unknown. Memmert S et, al conducted investigation under hypoxic and normoxic conditions, human primary periodontal ligament (PDL) fibroblasts $(\mathrm{n}=9$ ) were stimulated with Lipopolysaccharides (LPS) from Porphyromonas gingivalis (P.g.), a periodontal pathogenic bacterium. After different time points, cell viability 
was tested; p53 gene expression, protein synthesis, and activation were measured using quantitative RT-PCR, immunoblotting, and immunofluorescence. Also healthy and inflamed periodontal tissues were obtained from 12 donors to analyze p53 protein in oral inflammatory diseases by immunohistochemistry. Their result reviled that LPS-P.g. and hypoxia initially induced a significant upregulation of p53 mRNA expression and p53 protein levels. Conclusions which derived from their study are that p53 plays a pivotal role in PDL cell homeostasis and seems to be up regulated in oral inflammatory diseases. Upregulation of p53 may promote the destruction of periodontal integrity [13 ].

Zhou X et, al in their study focused on the effect of microRNA-138 (miR-138) as a potential regulator of periodontal stem cells as they affect homeostasis during inflammatory conditions. Their data indicate that miR-138 was significantly up regulated in our periodontal disease animal model. Also their data establish miR-138 inhibitor as a potential therapeutic agent for the prevention of the bone loss associated with advanced periodontal disease [14].

The researchers are still exploring a more suitable gene therapy approach for the use in the future for periodontal regeneration. Research shows that the bioactive materials are capable of stimulating periodontal healing. Among these materials, bioactive glass has been widely researched in periodontal regeneration. Cell therapy for periodontal regeneration is a new option. Currently, no effective regenerative measure for the destroyed periodontium is available, but tissue regeneration using stem cells has been a promising candidate for the purpose of periodontal regeneration [15].

\section{References}

1. Tsukamoto Y, Usui M, Yamamoto G, Takagi Y, Tachikawa T, Yamamoto $\mathrm{M}$, et al. Role of the junctional epithelium in periodontal innate defense and homeostasis. J Periodontal Res. 2012;47(6):750-757.

2. Darveau RP, Tanner A, Page RC. The microbial challenge in periodontitis. Periodontol. 1997;14(1):12-32.

3. Darveau RP. Periodontitis: a polymicrobial disruption of host homeostasis. Nat Rev Microbiol. 2010;8(7):481-490.
4. Bodet C, Andrian E, Tanabe S, Grenier D. Actinobacillus actinomycetemcomitans lypopolysaccharide regulates matrix metalloproteinase, tissue inhibitors of matrix metalloproteinase, and plasminogen activator production by human gingival fibroblasts: a potential role in connective tissue destruction. J Cell Physiol. 2007; 212(1):189-194.

5. Sekhon BS. Matrix metalloproteinases - an overview. Research and Reports in Biology. 2010;1:1-20.

6. Hannas AR, Pereira JC, Granjeiro JM, Tjдderhane L. The role of matrix metalloproteinases in the oral environment. Acta Odontologica Scandinavica. 2007; 65(1):1-13.

7. Giannobile, WV. Host-response therapeutics for periodontal diseases. J Periodontol. 2008;79(8S):1592-1600.

8. Leibbrandt A, Penninger JM. RANK/RANKL: regulators of immune responses and bone physiology. Ann N Y Acad Sci. 2008;1143: 123150. doi: 10.1196/annals.1443.016

9. Cochran DL. Inflammation and bone loss in periodontal disease. J Periodontol. 2008;79(8S):1569-1576.

10. Zhang S, Barros SP, Moretti AJ, Yu N, Zhou J, Preisser JS, et al. Epigenetic regulation of TNFA expression in periodontal disease. J Periodontol. 2013;84(11):1606-1616.

11.Willi M, Belibasakis GN, Bostanci N. Expression and regulation of triggering receptor expressed on myeloid cells 1 in periodontal diseases. Clin Exp Immunol. 2014;178(1):190-200.

12.Zhang F, Suzuki M, Kim IS, Kobayashi R, Hamada N, Sato F, et al. Transcription factor DEC1 is required for maximal experimentally induced periodontal inflammation. J Periodontal Res. 2018.

13. Memmert S, Gölz L, Pütz P, Jäger A, Deschner J, Appel T, et al. Regulation of p53 under hypoxic and inflammatory conditions in periodontium. Clin Oral Investig. 2016;20(7):1781-1789. doi: 10.1007/s00784-0151679-x

14.Zhou X, Luan X, Chen Z, Francis M, Gopinathan G, Li W, et al. MicroRNA-138 Inhibits Periodontal Progenitor Differentiation under Inflammatory Conditions. J Dent Res. 2016;95(2):230-237. doi: $10.1177 / 0022034515613043$

15. Diomede F, D’Aurora M, Gugliandolo A, Merciaro I, Ettorre V, Bramanti A, et al. A novel role in skeletal segment regeneration of extracellular vesicles released from periodontal-ligament stem cells. Int J Nano medicine. 2018;13:3805-3825. Doi: https://doi.org/10.2147/IJN. S162836 